\title{
Paraplegic Standing Supported by FES-Controlled Ankle Stiffness
}

\author{
Ralf-Peter Jaime, Zlatko Matjačić, and Kenneth J. Hunt
}

\begin{abstract}
The objective of this paper was to investigate whether a paraplegic subject is able to maintain balance during standing by means of voluntary and reflex activity of the upper body while being supported by closed loop controlled ankle stiffness using FES. The knees and hips of the subject were held in extended positions by a mechanical apparatus, which restricted movement to the sagittal plane. The subject underwent several training sessions where the appropriate level of stiffness around the ankles was maintained by the mechanical apparatus. This enabled the subject to learn how to use the upper body for balancing. After the subject gained adequate skills closed-loop FES was employed to regulate ankle stiffness, replacing the stiffness provided by the apparatus. A method to control antagonist muscle moment was implemented. In subsequent standing sessions, the subject had no difficulties in maintaining balance. When the FES support was withheld, the ability to balance was lost.
\end{abstract}

Index Terms-Ankle joint stiffness, FES, functional electrical stimulation, integrated voluntary control, multipurpose rehabilitation frame, paraplegic standing.

\section{INTRODUCTION}

\section{A. Motivation}

$\mathbf{R}$ ESTORING standing after spinal cord injury has a number of well-known therapeutic and functional benefits. Apart from overcoming several physiological problems caused by prolonged immobilization such as bladder infections, pressure sores, spasticity, and problems in maintaining blood pressure, regaining some functionality increases independence and, consequently, the quality of life for the affected people.

\section{B. Related Work}

Restoring standing by means of functional electrical stimulation (FES) after spinal cord injury has been a subject of research for many years. The first continuous FES-supported standing programme was initiated by Kralj and Bajd [1] in Ljubljana in 1979 and continues until the present day. The early approaches used open-loop stimulation to hold the knees extended. The hips

Manuscript received April 27, 2001. The work of R.-P. Jaime was supported by Grant HPMT-CH-00-00092-01 of the European Commission with a Marie Curie Fellowship for a research visit to the Center for Sensory-Motor Interaction at Aalborg University.

R.-P. Jaime was with the Centre for Systems and Control, University of Glasgow, Glasgow, Scotland, U.K. He is now with Robert Bosch GmbH, Germany (e-mail: ralf-peter.jaime@ de.bosch.com).

Z. Matjačić was with Center for Sensory-Motor Interaction, Aalborg University, Denmark. He is now with the Institute for Rehabilitation, Republic of Slovenia and the Institute Jožef Stefan, Ljubljana, Slovenia (e-mail: zlatko.matjacic@mail.ir-rs.si).

K. J. Hunt is with the Centre for Systems and Control, University of Glasgow, Glasgow, G12 8QQ, Scotland, U.K. (e-mail: k.hunt@mech. gla.ac.uk).

Digital Object Identifier 10.1109/TNSRE.2002.806830 were hyperextended while subjects used their arms to maintain balance. Due to fatigue of the artificially stimulated muscles, a paraplegic person is able to stand only for a few minutes. In order to prolong the achievable periods of standing, Kralj et al. [2] proposed posture switching. This allows the muscles to relax.

An upright human body is inherently unstable and is subject to unexpected disturbances. Thus, the application of feedback control was investigated by Jaeger [3]. He developed a simulation model of quiet standing to study the potential use of closed-loop stimulation orthoses in midthoracic spinal cord injury. It consisted of a second-order linear and time-invariant muscle dynamics followed by a single-link inverted pendulum and was regulated by a standard PID-controller. In a simulation study he found that, under biomechanical constraints and subject to the availability of torque produced by the stimulated muscle, it might be possible to restore quiet standing.

A more complicated simulation model of paraplegic standing was developed by Khang and Zajac [4], [5] involving nonlinear musculotendon dynamics and a planar three-segmental body dynamics. Arm movement was modeled as an external disturbance. Their goal was to design a feedback system that could restore and maintain vertical posture without intentional user interaction. Taking advantage of muscle redundancy, they developed an algorithm to distribute the net activation calculated by the feedback controller among all the muscles crossing a joint. This was motivated by the assumption that minimization of energy expenses reduces muscle fatigue. Their model included 13 muscles in the lower extremities. A static output gain controller was designed based on the linearised body dynamics and other approximations concerning the capabilities of the muscles. However, this approach does not take the residual sensory and motor function of the intact upper body into account. The simulation model was intended to be a "benchmark" for the development of future FNS systems. The approach was not experimentally tested with human subjects.

The first experimental attempt to apply feedback control to the task of paraplegic standing was undertaken by Hunt et al. [6], [7] in 1997. Their goal was to investigate the fundamental limitations of FES-supported paraplegic standing, while excluding external inputs from the CNS. Feedback control was applied to stabilize the body and no arm support was permitted. They used a cascade control structure to decouple the nonlinear and time-variant properties of the stimulated muscle from the task of stabilizing the body. The subject was assumed as a single-link inverted pendulum. All joints above the ankle joint were braced by a custom-made body shell. The body was leaning slightly forward and the plantarflexor muscles were stimulated. There 
was no switching between different muscle groups. An optimal control approach was chosen as control method for both the inner loop regulating the muscle moment [8] and the outer loop stabilizing the body. The reference moment provided by the outer loop controller was divided equally between the left and right legs. They found that, while an intact subject was able to stand for a considerable time, standing of the paraplegic subject (T5, 35 years of age, 13 years post-injury, male) was limited to around 30-40 s due to muscle fatigue and spasticity.

An improved control design methodology was proposed by Hunt et al. [9] providing a more reliable and robust design. The moment loop was now considered as a single-input/singleoutput system. The same stimulation was applied to the left and right leg so that the total moment was balanced between the left and right leg. It was now possible to deal with the often present strong asymmetry between the left and right leg in paraplegics. A pole-placement design was applied. This made the nominal closed-loop response independent of the nominal plant model. Experimental results with a paraplegic subject (T7/8, 44 years old, 4 years post-injury, male) showed that considerably longer periods of standing (up to several minutes) were possible [10].

All the previous control schemes cannot be called "functional" because either the subject has to use his arms to stabilize himself [1] or the subject is restricted in his freedom of movement by rigid and rather conservative simplifications [6], [7], [10].

Matjačić and Bajd [11], [12] have demonstrated that a paraplegic subject, after appropriate training, is able to stabilize himself using his trunk muscles if a certain level of stiffness around the ankle joint is present (approximately $10 \mathrm{Nm} / \mathrm{deg}$ ). The subject was assumed as a double-link inverted pendulum with the knees mechanically braced by an experimental apparatus and the trunk free to move voluntarily. The ankle stiffness was applied by hydraulic actuators, which acted as artificial ankle joints.

In [13] Matjačić suggests that a simple static stiffness model, neglecting higher-order effects, can adequately describe the postural responses following perturbations in the ankle and hip joints in neurologically intact persons. The stiffness values were found to be $17 \pm 5 \mathrm{Nm} / \mathrm{deg}$ for forward directed perturbations and $13 \pm 6 \mathrm{Nm} / \mathrm{deg}$ for backward directed perturbations.

Recently, Hunt et al. [14], [15] have investigated the control of paraplegic ankle joint stiffness using FES while standing. This approach incorporates moment control of antagonist muscles. One intact ( 29 years old, male) and one paraplegic person (T7/8, 44 years old, 4 years post-injury, male) participated in their study. They found that accurate ankle stiffness control can be achieved with FES but is fundamentally limited by the strength of the muscles. They concluded that ankle stiffness control has the potential to ease the task of stabilizing upright posture by application of additional upper-body forces.

Several studies have been undertaken to investigate the natural mechanism of standing in intact subjects [16]-[19]. In fact, research on this issue remains controversial [20], [21].

\section{Our Approach}

Our aim was to investigate the feasibility of "functional" paraplegic standing by means of FES. The approach was to combine the work from Matjačić and Bajd [11], [12] with the results from Hunt et al. [14], [15] and to substitute the hydraulic actuators by closed-loop FES to provide stiffness control at the ankles. There are several reasons why the potential of ankle stiffness control by FES needs to be studied:

- A higher bandwidth can be achieved using hydraulic actuators than using FES. Therefore, the stiffness controlled by means of FES can only be less accurate than the stiffness applied by hydraulic actuators.

- Any FES system is affected by fatigue and spasticity, while an "artificial" ankle joint actuated by a hydraulic system is not.

- A hydraulic system is a good experimental setup to study the feasibility of the principle but on the other hand is not useful as a daily life application. In comparison, FES provides more flexibility and independence, especially when it comes to an implanted system. Furthermore, FES has a greater potential to extend the system toward more functionality.

Mihelj et al. [22]-[24], in a parallel development, have also attempted to combine FES control of the ankle with voluntary upper-body input. Their work is based upon the control of the position of the Centre of Pressure relative to the ankle joint axis.

\section{METHODS}

\section{A. Subject}

The experiments reported here were performed with a paraplegic subject with a complete lesion at T5. The subject was male, 38 years of age, 8 years post-injury, and psychologically and physically in good condition. The subject gave informed consent for participation in the experimental procedure.

\section{B. Apparatus}

The device called the "multipurpose rehabilitation frame" (MRF) is described in detail in [25], [26]. The frame provides two degrees of freedom, i.e., sagittal and coronal planes. It supports the subject around the pelvis and permits motion in a range of $\pm 18^{\circ}$ around the vertical position in both planes. Two hydraulic actuators can independently control the frame in either of the two planes of motion. The actuators can be regarded as an artificial ankle joint and hip joints. The subject's feet can be positioned using cylindrical pegs on two aluminum blocks containing a grid of holes. The angle of inclination in both the sagittal and coronal planes is measured by a potentiometer. For the experiments presented in this paper the motion of the frame was restricted to the sagittal plane by a high value of stiffness in the coronal plane provided by the hydraulic servo system.

\section{Control Structure}

The frame was used to brace the knees and hips of the paraplegic subject and restrain the movement to the sagittal plane as shown in Fig. 1.

In this configuration, the subject is assumed as a double-link inverted pendulum. The subject in our study was not able to keep his upper body upright without holding on to the frame due to his rather high level of lesion (T5) and his rather weak trunk 


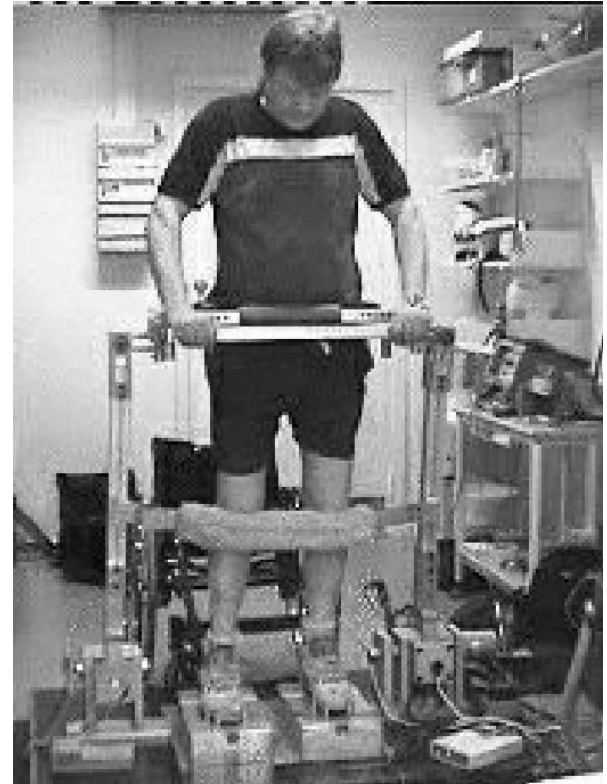

Fig. 1. The paraplegic subject is balancing while standing in the frame.The subject in our study was forced to hold onto the frame with his arms due to his weak trunk muscles.

muscles. But since the frame is moving with the subject's lower body, this does not prevent the subject from falling over and requires active balancing to maintain standing.

While the lower body-half was supported by closed-loop FES the upper body-half was under voluntary control by the CNS. A strategy to control the stiffness around the ankle joints was implemented incorporating closed-loop moment tracking of the antagonist plantarflexor and dorsiflexor muscles. The subject was standing on two AMTI-forceplates OR6-5-1000 measuring the ground reaction forces and moments at the axes of the orthogonal coordinate system. This allowed us to independently control the muscle moment in the left and right ankles. Practical sensors for ankle moment feedback are still an open topic. Further experiments shall investigate the benefit of moment feedback [27]. Fig. 2 illustrates the experimental situation.

The angle of inclination of the frame in the sagittal plane $\theta_{S}$ was measured and multiplied by the desired stiffness value (typically $10 \mathrm{Nm} / \mathrm{deg}$ ). This provided the total reference moment $m_{\text {ref,total }}$. The total reference moment was distributed between the left and right ankles following the relative load distribution according to (1) and (2)

$$
\begin{aligned}
& m_{\mathrm{ref}, 1}=\frac{F_{z, l}}{F_{z, l}+F_{z, r}} m_{\text {ref,total }} \\
& m_{\text {ref,r }}=\frac{F_{z, r}}{F_{z, l}+F_{z, r}} m_{\text {ref,total }}
\end{aligned}
$$

where $F_{z, l}$ and $F_{z, r}$ are the vertical components of the ground reaction force measured by the left and right forceplates.

In the ideal case when the transfer function from $m_{\text {ref,total }}$ to $m_{\text {total }}$ in Fig. 2 is unity, the configuration of Fig. 2 has been analyzed in great detail in [11]. The block labeled "desired stiffness" can be regarded as a simple proportional controller. From [11] it becomes clear that P-control alone is not sufficient to stabilize the double-link inverted pendulum. The idea behind this setup can be summarized as follows: a certain ankle stiffness makes stable standing easier while the task of stabilizing is left to the paralyzed subject, utilizing his residual motor-sensory abilities.

The blocks labeled "left ankle" and "right ankle" in Fig. 2 are each closed-loop moment controllers. Fig. 3 gives a deeper insight into their structure.

The block $C_{m}^{p, d}$ represents two moment controllers for plantarflexor and dorsiflexor dynamics with common states and a suitable scheduling strategy. Thus, there are four moment controllers in total. The controllers $C_{m}^{p, d}$ determine a control signal $u$ depending on the error between the reference moment $m_{\text {ref }}$ and the measured moment $m_{\text {meas }}$. The sign of the control signal $u$ determines whether it is applied to the plantarflexor $\left(u^{\text {plan }}\right)$ or the dorsiflexor $\left(u^{\text {dorsi }}\right)$ muscles. The stimulator transforms the control signal into electrical pulses of a corresponding pulsewidth $p w^{\text {plan }}$ or $p w^{\text {dorsi }}$.

For stimulation of the muscles we used an eight-channel programmable stimulator known as the "Stanmore Stimulator" described in [28]. It is driven by a PC via the serial port and produces current controlled monophasic rectangular pulses up to a duration of $800 \mu \mathrm{s}$. The amplitude of the pulses is adjustable in steps of $10 \mathrm{~mA}$ and the pulsewidth is adjustable in steps of $2 \mu \mathrm{s}$. The stimulator was operated at a constant frequency of $20 \mathrm{~Hz}$ (sampling interval $50 \mathrm{~ms}$ ). All experimental procedures were based on MATLAB/SIMULINK. Data acquisition and real-time control were done using the Real-time Toolbox. ${ }^{1}$ The Polynomial Toolbox ${ }^{2}$ was employed for the controller design. We used round surface electrodes, with a diameter of 2 inches, placed over the gastrocnemius muscle for plantarflexion and the tibialis anterior muscle for dorsiflexion.

\section{Session History}

The following sessions were carried out in the course of this study.

- Three sessions of balance training. First, the subject underwent several training sessions where the appropriate level of stiffness around the ankles was maintained by the frame. This enabled the subject to learn how to use the upper body for balancing at a stiffness level of $8 \mathrm{Nm} / \mathrm{deg}$. The subject gained adequate balancing skills after three sessions of balancing that lasted up to half an hour. FES was introduced after the initial three sessions.

- One session of plantarflexor moment control (i.e., "Identification" and "Test M").

- Two sessions of standing under FES-controlled ankle stiffness (i.e., "Identification," "Test M," and "Test B"). The results presented in this paper are from the second standing session.

\section{E. Experimental Procedure}

The experimental procedures can be summarized as follows (Steps 2 and 3 are similar to [9]:

\footnotetext{
${ }^{1}$ Www.humusoft.com.

${ }^{2}$ www.polyx.com.
} 


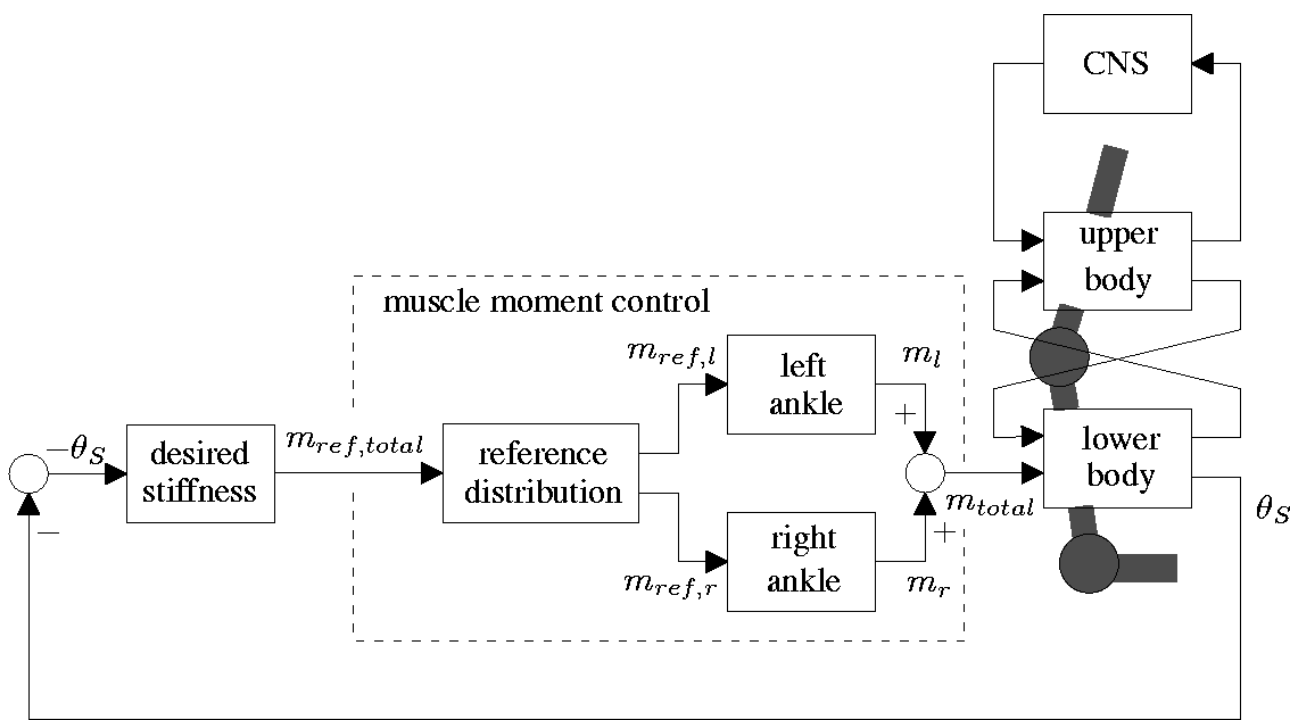

Fig. 2. Block diagram of the ankle stiffness control and standing strategy. The blocks denoted "left ankle" and "right ankle" are closed-loop controllers for the left and right ankle moment, respectively.

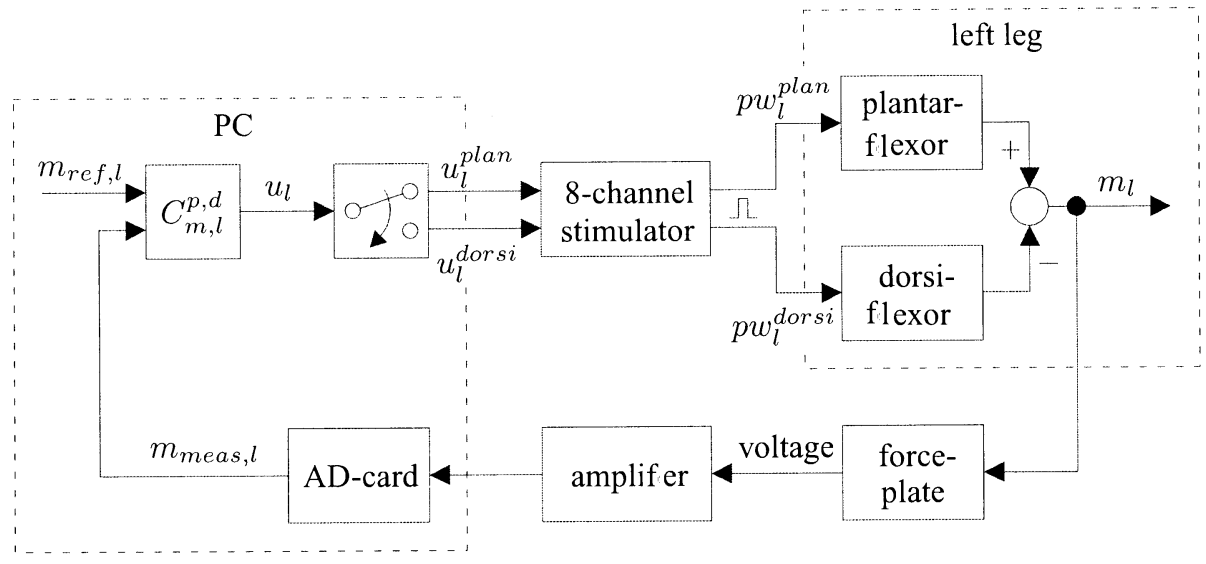

Fig. 3. Closed-loop moment control for left ankle. The moment control for the right side is identical, with the index "l" replaced by "r." Each of the blocks labeled "left ankle" and "right ankle" in Fig. 2 have this structure.

1) Identification. The paraplegic subject stood on two AMTI forceplates, having knees mechanically locked by a leather belt as shown in Fig. 1. During the identification procedure the frame was fixed by an aluminum bar mounted on the ceiling in order to ensure static conditions and to gain better results from the identification procedure. The subject was asked to stand still. The following tests were carried out separately for the plantarflexor and dorsiflexor muscles of both the left and right legs.

a) Test $\mathbf{C}$. The purpose of this test is to establish a suitable amplitude of the stimulation pulses, since we used pulses with a constant amplitude and a varying pulsewidth. Starting with an appropriate value of current the pulsewidth was ramped up from $50-500 \mu \mathrm{s}$ in $5 \mathrm{~s}$ and the produced moment was measured. This pattern was repeated with a different amplitude of the current if necessary. The stimulation amplitude was set to $60 \mathrm{~mA}$ for all four muscles during the further course of the experiment.

b) Test PRBS. This test is an open-loop test using a stimulation signal where the pulsewidth has a $\mathrm{PRBS}^{3}$ form. The produced moment was measured. The PRBS signal

${ }^{3}$ Pseudo-Random Binary Sequence could be applied around a range of mean stimulation pulsewidth levels. The amplitude of the PRBS signal was set at $35 \mu \mathrm{s}$. The PRBS signal was designed off-line to excite the major dynamic properties of the muscle. It has a period of 155 samples and is constant for at least 5 samples after each transition [29].

The input/output data gained from the PRBS test were used to identify a local linear transfer function at the stimulated operation point for each muscle group. Following the identification procedure and assessment of the quality of the identified models a moment controller was designed for each muscle group. The control design was judged on the basis of the closed-loop frequency responses before testing.

2) Test M. This is a test of closed-loop moment tracking. It was carried out to check whether the moment feedback loop was well designed and working properly before a series of standing test would be carried out.

3) Test B. The aluminum bar fixing the frame was removed. The hydraulic circuit of the frame was switched on. The frame provided support in the coronal plane by a stiffness of $10 \mathrm{Nm} / \mathrm{deg}$ in order to restrain the movement to 

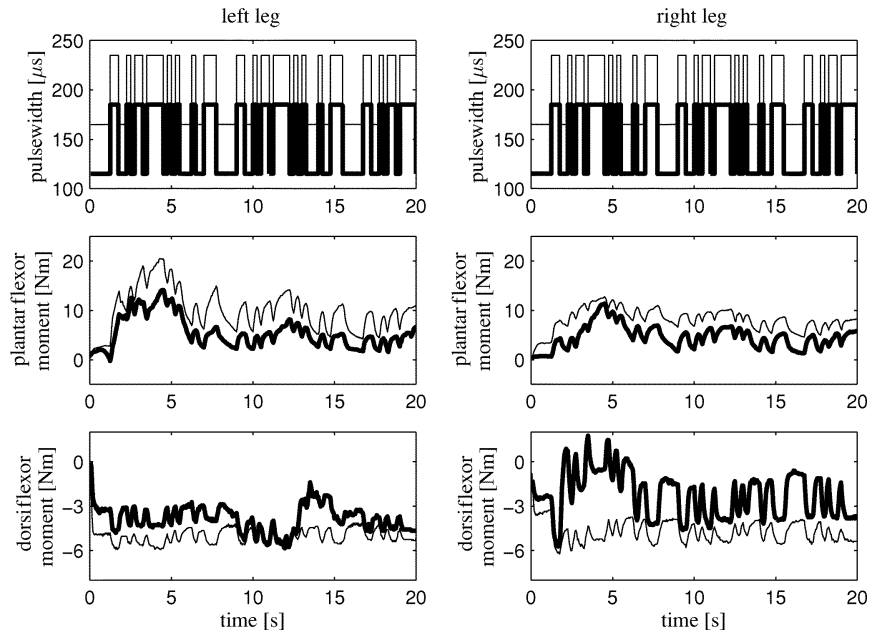

Fig. 4. Results of Test PRBS. The bold lines in the moment plots correspond to the bold line in the pulsewidth plot. The two PRBS signals for each side were applied to both muscle groups on that side, hence, four moment plots for each side are shown.

the sagittal plane. In the sagittal plane the frame provided a stiffness of $2 \mathrm{Nm} / \mathrm{deg}$ in order to compensate the load imposed by the weight of the frame. The frame was held in the upright position by the experimenter and, after the stimulation was switched on, released while the subject was under closed-loop FES-controlled ankle stiffness. In order to maintain standing the subject was forced to balance actively. The results are presented in chronological order. The first successful standing trial is shown and the quality of stiffness control is analyzed (cf. Figs. 7-10) Subsequent standing trials show clearly a learning effect in the patient's skills to deal with the studied control scheme (Figs. 11-15). Even periods of quiet standing could be achieved (cf. Fig. 12, Fig. 13). Finally, the stabilizing contribution of the FES-controlled ankle stiffness is investigated (cf. Fig. 14, Fig. 15).

\section{EXPERIMENTAL RESULTS}

\section{A. Test PRBS}

Results of Test PRBS are presented in Fig. 4. The test was carried out for a mean level of $150 \mu$ s (bold) and once repeated for a mean level of $200 \mu$ s (thin). It lasted for $20 \mathrm{~s}$. The stimulation was then not further increased because we knew by experience that the muscles have a higher dc-gain at low stimulation levels. This is a result of the inverse recruitment pattern by artificial stimulation. The identified model with the higher dc-gain would later be used for the controller design. The first 5 $\mathrm{s}$ of the test were omitted when performing the estimation of the transfer function to exclude the transient response at the beginning of each stimulation cycle. There are also disturbances to be seen in the muscle response. They can be a result of spasticity or an influence of the upper body. This observation emphasises the importance of fixing the frame during the identification process.

\section{B. Identification}

Based on the input/output data from Test PRBS we identified two local linear time-invariant transfer functions for each
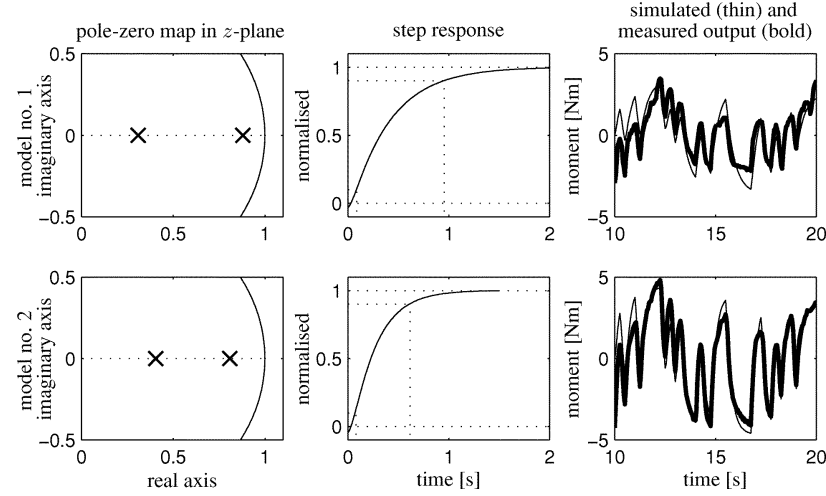

Fig. 5. Results identification plantarflexor left. The results for the other muscle groups are similar in nature.

TABLE I

SUMMARY OF THE IDENTIFIED LOCAL MODELS

\begin{tabular}{|cc|c|c|c|c|}
\hline muscle & & model no. & rise time [s] & Dc-gain [Nm/ $\mu$ s] & RMSE [\%] \\
\hline plantarflexor left & & 1 & 0.87 & 0.093 & 7.1 \\
& $\Rightarrow$ & $\mathbf{2}$ & $\mathbf{0 . 5 4}$ & $\mathbf{0 . 1 2 0}$ & $\mathbf{3 . 7}$ \\
\hline dorsiflexor left & $\Rightarrow$ & $\mathbf{1}$ & $\mathbf{0 . 7 3}$ & $-\mathbf{0 . 0 3 5}$ & $\mathbf{1 5 . 8}$ \\
& & 2 & 0.41 & -0.019 & 3.5 \\
\hline plantarflexor right & $\Rightarrow$ & $\mathbf{1}$ & $\mathbf{0 . 7 4}$ & $\mathbf{0 . 0 8 4}$ & $\mathbf{5 . 5}$ \\
& & 2 & 0.58 & 0.060 & 3.5 \\
\hline dorsiflexor right & $\Rightarrow$ & $\mathbf{1}$ & $\mathbf{0 . 2 2}$ & $\mathbf{- 0 . 0 4 3}$ & $\mathbf{5 . 5}$ \\
& & 2 & 0.38 & -0.023 & 3.5 \\
\hline
\end{tabular}

muscle group using the least squares criterion. We identified second-order models $G_{p}\left(q^{-1}\right)$ of the following form:

$$
G_{p}\left(q^{-1}\right)=\frac{b_{0} q^{-1}}{1+a_{1} q^{-1}+a_{2} q^{-2}}
$$

Here, $q^{-1}$ is the delay operator. The results of the estimation process are shown in Fig. 5 for the left plantarflexor muscles. The results for the other muscle groups are similar in nature. The pole locations, the normalized step response and a comparison between the simulated and measured output are presented. Model no. 1 corresponds to the lower stimulation level highlighted by the bold lines in the plots of Test PRBS. It can be seen that the resulting models are dominantly first-order and the simulated model response fits well enough to the measured data. The results are summarized in Table I, where rise time and dc gain as well as the relative mean square error (rmse) between the simulated and the measured output are given for each model. The models that were finally chosen for the controller design are highlighted in Table I. Those are the ones with the highest absolute value of the dc gain.

\section{Control Design}

The nominal muscle transfer functions are summarized in Table II. The four moment controllers were designed by pole-assignment [30]. A two degree-of-freedom design was chosen since we were mainly interested in reference tracking. The design parameters were specified in terms of the rise time and damping. The rise time for the control response was chosen as $0.5 \mathrm{~s}$ for the left plantarflexors, $0.7 \mathrm{~s}$ for the left dorsiflexors as well as for the right plantarflexors and $0.3 \mathrm{~s}$ for the right dorsiflexors. The rise time for reference tracking was specified as 
TABLE II

NOMINAL MODELS FOR CONTROL DESIGN

\begin{tabular}{|c|l|}
\hline muscle & \multicolumn{1}{|c|}{ transfer function } \\
\hline plantarflexor left & $G_{p}^{p l}\left(q^{-1}\right)=\frac{0.0137 q^{-1}}{1-1.213 q^{-1}+0.327 q^{-2}}$ \\
dorsiflexor left & $G_{p}^{d l}\left(q^{-1}\right)=\frac{-0.00336 q^{-1}}{1-1.175 q^{-1}+0.272 q^{-2}}$ \\
plantarflexor right & $G_{p}^{p r}\left(q^{-1}\right)=\frac{0.00717 q^{-1}}{1-1.251 q^{-1}+0.336 q^{-2}}$ \\
dorsflexor right & $G_{p}^{d r}\left(q^{-1}\right)=\frac{-0.00839 q^{-1}}{1-1.221 q^{-1}+0.414 q^{-2}}$ \\
\hline
\end{tabular}
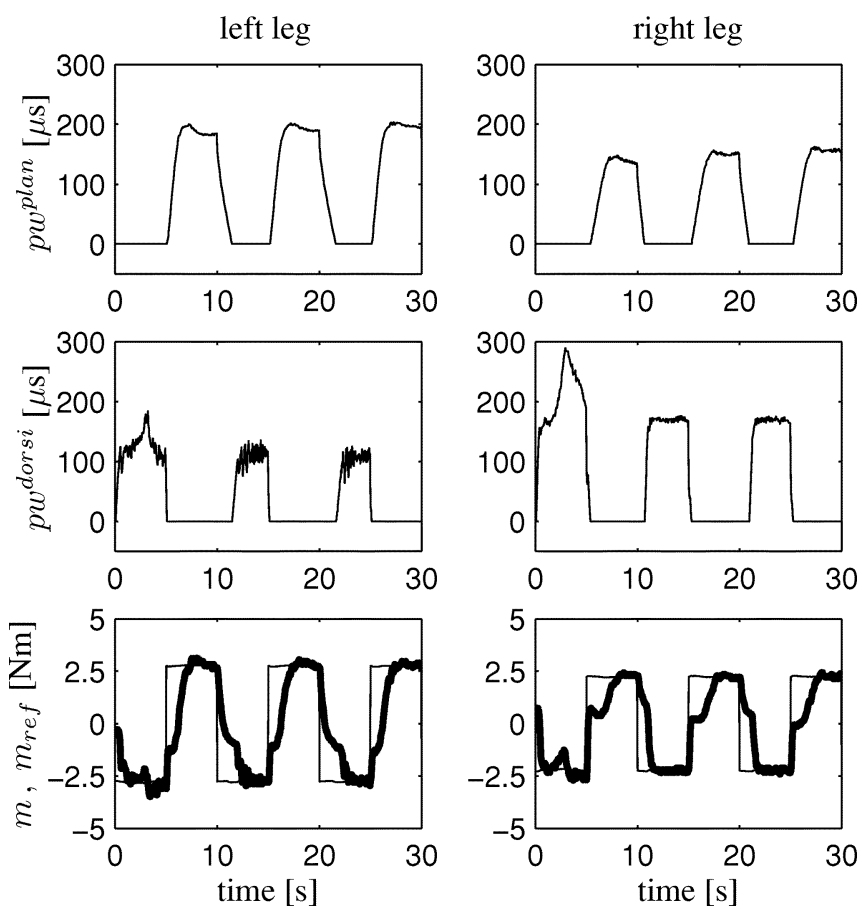

Fig. 6. Moment reference tracking test.

$0.2 \mathrm{~s}$ for each of the four moment controllers. All damping factors were set to 1 . The design procedure is outlined in detail in [9].

\section{Test $M$}

The result of typical moment controller tests are shown in Fig. 6. The plots in the upper row show the plantarflexor stimulation pulsewidth. The dorsiflexor stimulation signal is presented in the middle row. The plots in the row below show the reference moment (thin line) and the controlled moment (thick line). The reference moment was a $\pm 5 \mathrm{Nm}$ square wave signal that was distributed among the left and right leg according the current load distribution. It can be seen from the left leg moment plot that switching between plantarflexor and dorsiflexor muscles occurs at a slightly negative moment. Therefore, the moment step required from the plantarflexor muscles is higher than the moment step required from the dorsiflexor muscles. This explains why a higher stimulation is required from the plantarflexors than from the dorsiflexors although the plantarflexors are stronger than the dorsiflexors.
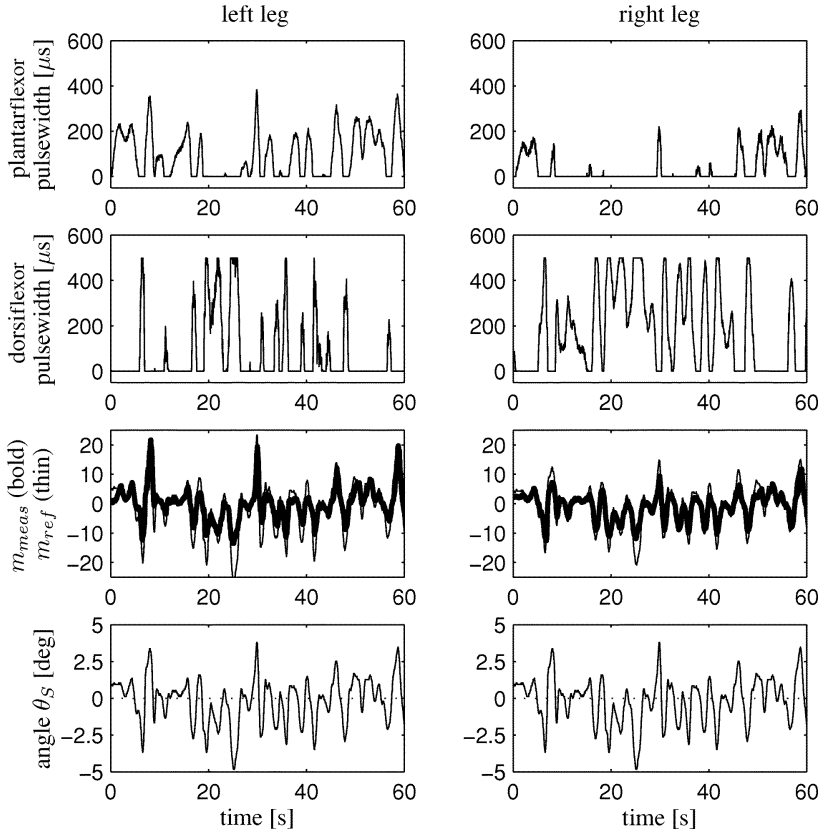

Fig. 7. First successful standing trial. Specified stiffness $k_{s}=10 \mathrm{Nm} / \mathrm{deg}$. The angle plots are the same for the left and right sides.

The design for the plantarflexor muscles is slightly conservative both for the left and right leg but this was improved by implementing a fast prefilter during the further standing tests.

\section{E. Test B}

A typical standing trial is shown in Fig. 7. After the stimulation was switched on the subject was released and was balancing on his own under the influence of the FES controlled ankle stiffness. The subject was asked to keep balance as learned during the training sessions when stiffness was provided by the hydraulic actuators. The stiffness was specified as $10 \mathrm{Nm} / \mathrm{deg}$. The frame provided a stiffness of $10 \mathrm{Nm} / \mathrm{deg}$ in the frontal plane (restraining movement only to the sagittal plane) and $2 \mathrm{Nm} / \mathrm{deg}$ in the sagittal plane in order to compensate the load imposed by the weight of the frame.

The plots at the bottom (fourth row) show the angle of inclination of the frame, i.e., the lower body $\theta_{S}$ (cf. Fig. 2). The plots above (third row) present the reference moment $m_{\text {ref }}$ (thin line) and actual measured moment $m_{\text {meas }}$ for the left and right leg (thick line). The upper plots show the stimulation signal of the plantarflexors $p w^{\text {plan }}$ (first row) and the dorsiflexors $p w^{\text {dorsi }}$ (second row).

The important result is that the angle of inclination remained limited to $\pm 5^{\circ}$ over the entire duration of the test, indicating the ability of the subject to successfully balance for the duration of one minute.

The time history of the actual achieved stiffness $k_{s}^{*}(t)$

$$
k_{s}^{*}(t)=\frac{m_{\text {total }}(t)}{\theta_{S}(t)}, \text { with } t=k T_{s}
$$

is shown in Fig. 8. In (4), $T_{s}$ is the sample time and $k$ is the sample instant.

The dots mark the calculated stiffness at the sampled time instances. The measured signals of moment and angle were 


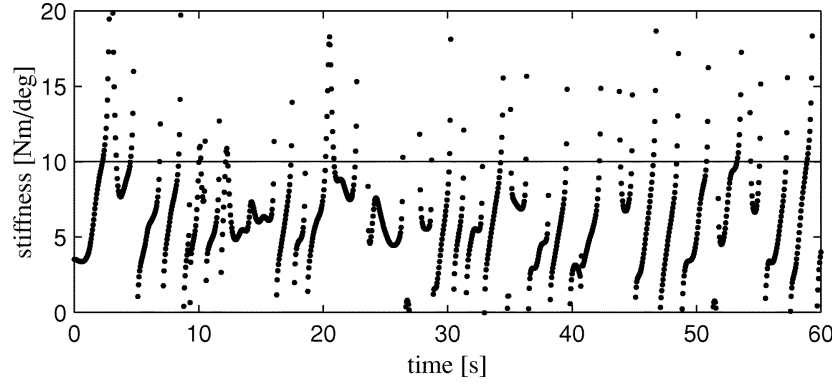

Fig. 8. Controlled stiffness during standing trial.

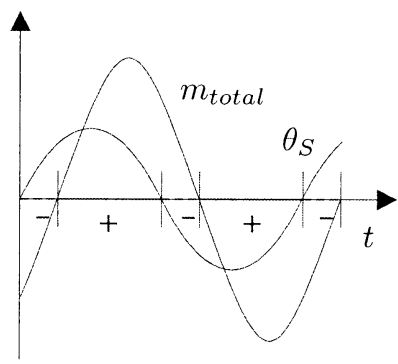

Fig. 9. Schematic representation of the signals which determine the actual stiffness $k_{s}^{*}$ when the subject is dynamically balancing. The "plus" and "minus" sign indicate the sign of the resulting stiffness.

zero-phase-shift digitally filtered by a Butterworth filter of tenth order with a cut-frequency of $1 \mathrm{~Hz}$ in order to eliminate the noise from the data. The accuracy of the achieved stiffness is limited by the closed-loop bandwidth of the moment control. With

$$
m_{\text {total }}(t)=\tilde{F}\left(q^{-1}\right) m_{\text {ref }}(t) \text { and } m_{\text {ref }}(t)=k_{s}(t) \theta_{S}(t)
$$

(4) becomes

$$
k_{s}^{*}(t)=\tilde{F}\left(q^{-1}\right) k_{s}(t)
$$

where $\tilde{F}\left(q^{-1}\right)$ is the transfer function for reference tracking of the moment loop. Due to the limited bandwidth of $\tilde{F}\left(q^{-1}\right)$ the total moment $m_{\text {total }}$ is slightly delayed in comparison to the angle signal $\theta_{S}$ when the subject is dynamically balancing (cf. Fig. 9).

Therefore, at the time instant when $m_{\text {total }}=0$ and $\theta_{S} \neq 0$ the stiffness $k_{s}^{*}=0$. On the other hand, when $\theta_{S}=0$ and $m_{\text {total }} \neq 0$ then the stiffness $k_{s}^{*} \rightarrow \infty$. There are also short periods when the stiffness is negative (negative values are cut off in Fig. 8, but cf. Fig. 9). This gives the calculated stiffness the uneven appearance.

Another representation of the achieved stiffness is shown in Fig. 10 where the stiffness is shown in the phase plane as moment versus angle. The reference stiffness of $10 \mathrm{Nm} / \mathrm{deg}$ is given by the straight dashed line while the straight solid indicates a stiffness of $6 \mathrm{Nm} / \mathrm{deg}$ around which the real stiffness approximately centers.

Another standing trial is shown in Fig. 11. The dotted lines in the angle plot emphasise the decreasing amplitude of the sway angle $\theta_{S}$. This suggests that the subject is learning to balance better during the experiment.

A third standing trial is shown in Fig. 12. The stiffness was reduced to $8 \mathrm{Nm} / \mathrm{deg}$. The learning process can also be observed

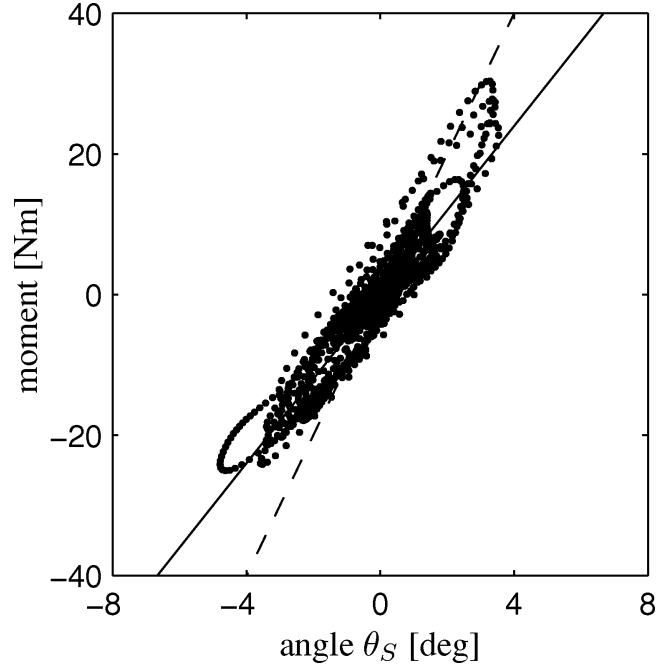

Fig. 10. Stiffness plot in phase plane. Straight dashed line: $10 \mathrm{Nm} / \mathrm{deg}$, straight solid line: $6 \mathrm{Nm} / \mathrm{deg}$.
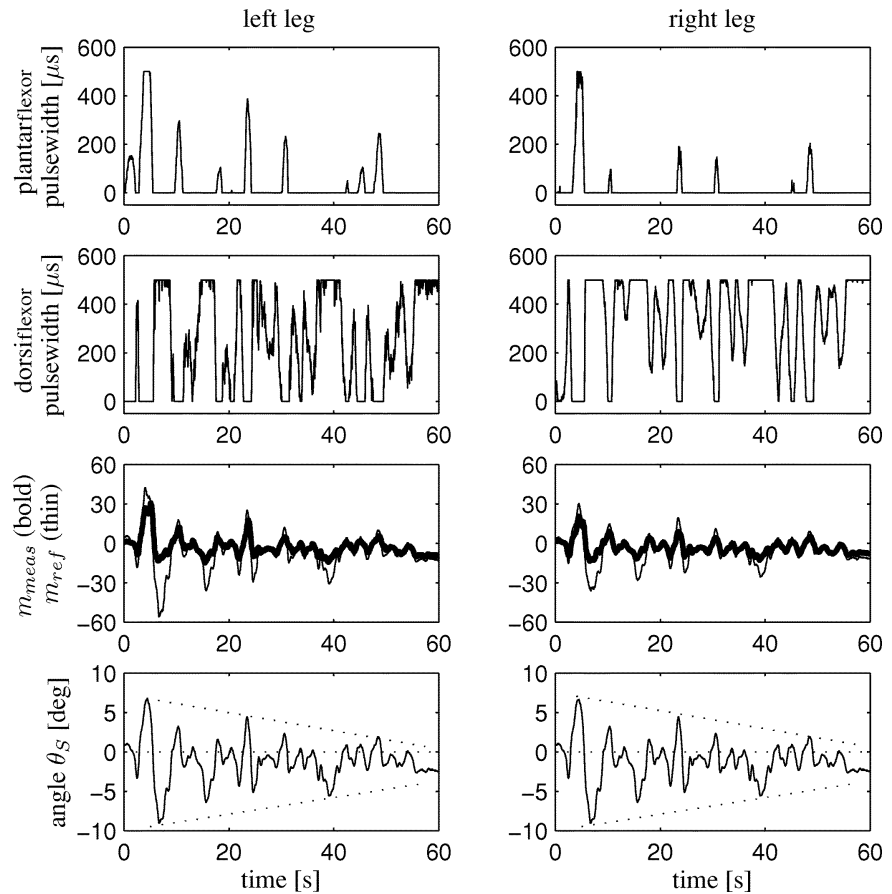

Fig. 11. Subsequent standing trial. The decreasing amplitude of the sway angle is emphasized by the dotted lines in the angle plots. This indicates a learning effect.

for the first $40 \mathrm{~s}$ during this trial. The subject even stood still for a few seconds between $t=35 \ldots 42 \mathrm{~s}$, approximately.

It can be seen from Fig. 13 that during the period of static standing $(t=35 \ldots 42 \mathrm{~s})$, the specified stiffness of $8 \mathrm{Nm} / \mathrm{deg}$ is actually achieved. The more static the standing the better the stiffness control.

A final standing trial is shown in Fig. 14. Here, the stimulation was suddenly switched off after $30 \mathrm{~s}$ while the subject was balancing. This was done in order to emphasize the stabilizing contribution of the FES-controlled stiffness. After the stimulation was switched off the subject immediately fell over. He was then set back by the experimenter into a nearly vertical position and fell again. This was repeated three times in the remaining 

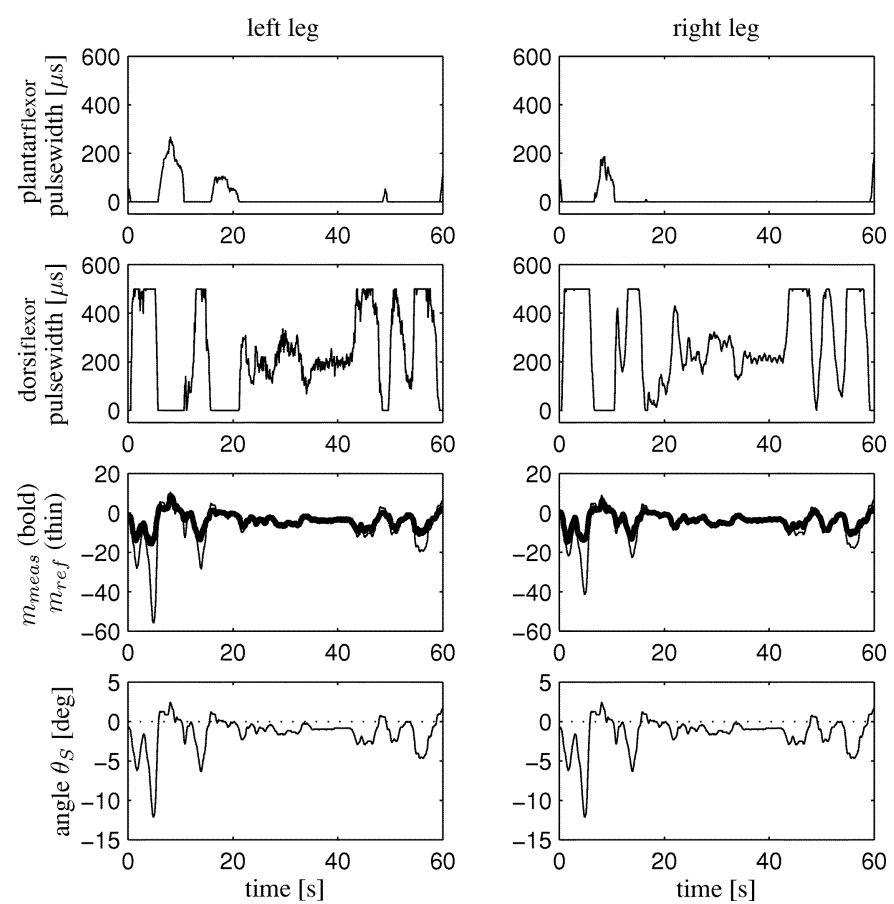

Fig. 12. Standing trial. The specified stiffness was reduced to $8 \mathrm{Nm} / \mathrm{deg}$ in order to avoid permanent saturation of the control signal. Note, the subject was trained to balance at a stiffness of $8 \mathrm{Nm} / \mathrm{deg}$.

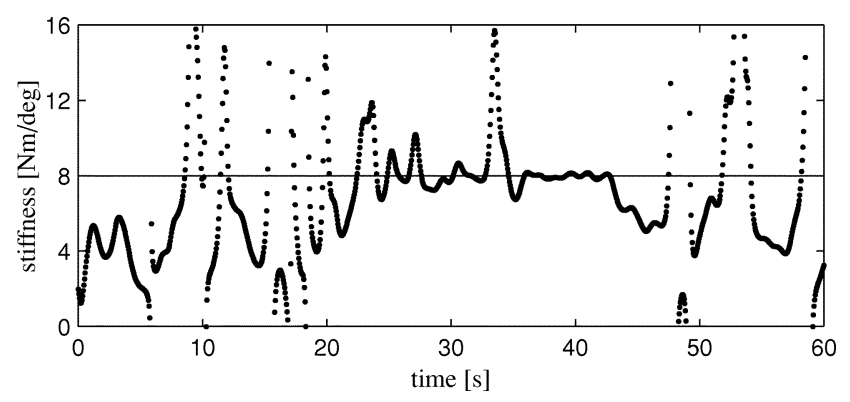

Fig. 13. Controlled stiffness during standing trial (corresponds to Fig. 12). For static conditions $(t=35 \ldots 42 \mathrm{~s})$, the specified stiffness can be achieved.

$30 \mathrm{~s}$ of this standing trial but the subject was not able to stand without stimulation. The stiffness was specified as $8 \mathrm{Nm} / \mathrm{deg}$.

Fig. 15 clarifies what happened at the stiffness level. Just before the stimulation was switched off the specified stiffness has been achieved but fell immediately to about $2 \mathrm{Nm} / \mathrm{deg}$ when it was switched off. The remaining stiffness seems to be the inherent stiffness of the ankle joints. Note, that the moment produced by the hydraulic actuators was not measured.

\section{DISCUSSION AND CONCLUSION}

The results have shown that paraplegic standing can be achieved by implementing FES-controlled ankle stiffness when the residual sensory-motor abilities of the patient are utilized. The subject in our study had to support his trunk by holding onto the frame due to his rather high level of lesion and his weak trunk muscles. A subject with a lower lesion and adequate trunk muscle strength should be able to perform the balancing task by using his trunk muscles alone and thus leaving the arms to perform a functional task. The results demonstrate the feasibility of stable paraplegic standing, when supported by
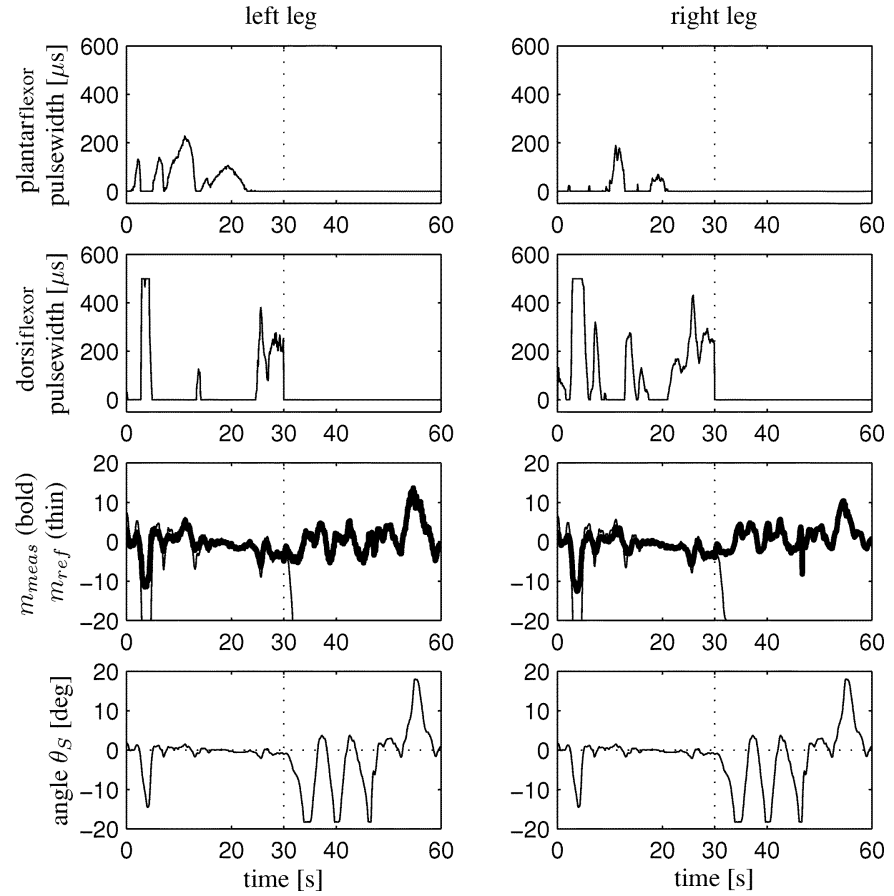

Fig. 14. Standing trial to emphasise contribution of FES. The stimulation was switched off after $30 \mathrm{~s}$.

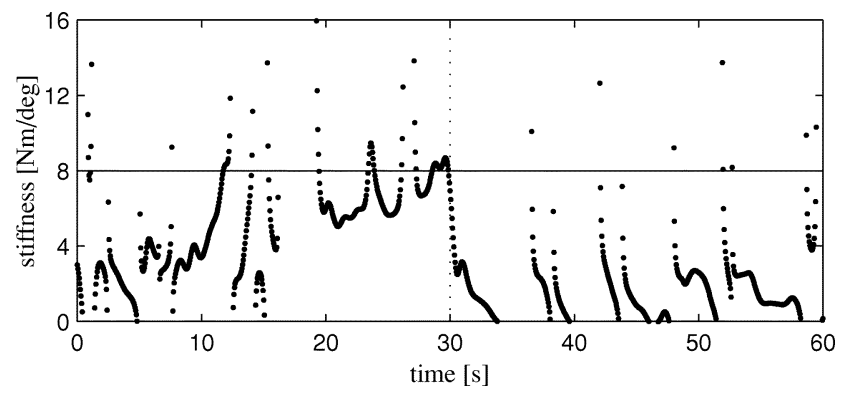

Fig. 15. Controlled stiffness during standing trial when stimulation was switched off after $30 \mathrm{~s}$ (cf. Fig. 14).

FES-controlled ankle stiffness. FES-controlled ankle stiffness makes an essential contribution to the overall control scheme and enables the patient to stand. This implies that when the patient's residual abilities are adequately trained, quite simple FES control strategies can be sufficient for stable standing. The results of this study can be summarized as follows:

Paraplegic standing can be achieved by FES-controlled ankle stiffness.

The subject learns to stand by means of the FES-controlled ankle stiffness. This learning process can be observed in the course of the presented results. Fig. 7 was the first standing trial during the session on which the results are based. The sway angle is rather restless for the entire duration of the trial. In the following trials, the subject settled more or less after some initial sways. In Fig. 11 the sway amplitude steadily decreased and came to rest during the trial shown in Fig. 12 and Fig. 14 before the stimulation was switched off. The same observation could be made during the trials which are not presented in the paper.

The accuracy of stiffness control is fundamentally limited by the bandwidth of the moment controller and the strength of the muscles. Clearly, when the controller saturates and the muscles 
are not able to produce the requested moment this will result in an underachievement with regard to the stiffness control. However, accurate stiffness control can be achieved in static conditions, i.e., when the subject is standing quietly enough.

Standing is also possible even when the specified stiffness can not be reached.

Mostly dorsiflexor stimulation was involved. It seems the subject preferred a posture where the lower body was slightly leaning backward while the upper body was leaning forward. Therefore it made sense to have a controller based on the dorsiflexor dynamics as well as the plantarflexor dynamics.

Altogether the subject was able to stand for a considerable time span. Ten standing trials were performed during the session each with a duration of one minute.

Requirements within the experiment were to be quick during identification and control design process in order to save muscle force for standing. However, the identification process has a great potential for automation and the control design method is simple and straightforward. The identification and control design process took approximately 10-15 $\mathrm{min}$. The whole experimental session lasted $1 \mathrm{~h}$.

No model of the biomechanical structure was required at all. The stiffness value was chosen using the results from [12]. This considerably simplifies the design process of the FES system since an accurate model of the biomechanical structure is hard to gain.

Stiffness is not sufficient to stabilize the body [9]. The idea was to make the task of stabilizing the erect body possible for the subject to do it himself using his own sensory-motor abilities. However, the FES-controlled ankle stiffness clearly contributes to the task of stabilizing the body.

\section{FUTURE WORK}

There are several issues open for future work.

- First, we plan to incorporate closed-loop control of the hip stiffness by using FES of abductor muscles. This could provide stability in the coronal plane and, furthermore, enable posture switching from one leg to the other in order to reduce fatigue and extend the periods of standing.

- Second, the current results of standing by FES-controlled stiffness shall be extended to add a viscosity term to the "pure" stiffness. This can easily be done by a feedback proportional to the angle velocity of the lower body and is expected to have a further stabilizing effect.

- Third, FES of the knee extensor muscle shall be included.

Altogether, this could lead to paraplegic standing completely relying on FES based on a simple and easy-to-use control scheme without the need of a model of the biomechanical structure of standing.

\section{ACKNOWLEDGMENT}

We would like to thank our subject for his cooperation in this study. Also, we thank C. Schmidt for proofreading the manuscript.

\section{REFERENCES}

[1] A. Kralj and T. Bajd, Functional Electrical Stimulation: Standing and Walking after Spinal Cord Injury. Boca Raton, FL: CRC, 1989.

[2] A. Kralj, T. Bajd, R. Turk, and H. Benko, "Posture switching for prolonging functional electrical stimulation standing in paraplegic patients," Paraplegia, vol. 24, pp. 221-230, 1986.

[3] R. J. Jaeger, "Design and simulation of closed-loop electrical stimulation orthoses for restauration of quiet standing in paraplegia," J. Biomechan., vol. 19 , pp. 825-835, 1986 .

[4] G. Khang and F. E. Zajac, "Paraplegia standing controlled by functional neuromuscular stimulation: Part I-computer model and control-system design," IEEE Trans. Biomed. Eng., vol. 36, pp. 873-884, Sept. 1989.

[5] G. Khang and F. E. Zajac, "Paraplegia standing controlled by functional neuromuscular stimulation: Part II-computer simulation studies," IEEE Trans. Biomed. Eng., vol. 36, pp. 885-894, Sept. 1989.

[6] K. J. Hunt, M. Munih, and N. Donaldson, "Feedback control of unsupported standing in paraplegia-Part I: optimal control approach," IEEE Trans. Rehabil. Eng., vol. 5, pp. 331-340, Dec. 1997.

[7] M. Munih, N. Donaldson, K. J. Hunt, and F. M. D. Barr, "Feedback control of unsupported standing in paraplegia-Part II:experimental results," IEEE Trans. Rehabil. Eng., vol. 5, pp. 331-340, Dec. 1997.

[8] K. J. Hunt, M. Munih, N. Donaldson, and F. M. D. Barr, "Optimal control of ankle joint moment: toward unsupported standing in paraplegia," IEEE Trans. Automat. Contr., vol. 43, pp. 819-832, June 1998.

[9] K. J. Hunt, H. Gollee, R. Jaime, and N. Donaldson, "Design of feedback controllers for paraplegic standing," Proc. IEEE Contr. Theory Applicat., vol. 148, pp. 97-108, Mar. 2001.

[10] H. Gollee, K. J. Hunt, D. Wood, and C. McFadden, "New results in feedback control of unsupported standing in paraplegic," in Proc. 6th Ann. Conf. Int. Functional Electrical Stimulation Soc., Cleveland, OH, 2001.

[11] Z. Matjačić and T. Bajd, "Arm-free paraplegic standing-Part I: control model synthesis and simulation," IEEE Trans. Rehabil. Eng., vol. 6, pp. 125-138, June 1998.

[12] Z. Matjačić and T. Bajd, "Arm-free paraplegic standing-part II: experimental results," IEEE Trans. Rehabil. Eng., vol. 6, pp. 139-150, June 1998.

[13] Z. Majačić, "Control of ankle and hip joint stiffness for arm-free standing in paraplegia," Neuromodulation, vol. 4, no. 1, pp. 37-46, 2001.

[14] K. J. Hunt, R.-P. Jaime, H. Gollee, and N. Donaldson, "Control of ankle joint stiffness while standing," in Proc. 5th Ann. Conf. Int. Functional Electrical Stimulation Soc., Aalborg, Denmark, 2000.

[15] K. J. Hunt, H. Gollee, and R.-P. Jaime, "Control of paraplegic ankle joint stiffness using FES while standing," Med. Eng. Phys., pt. 541, vol. 23, p. $555,2001$.

[16] R. C. Fitzpatrick, J. L. Taylor, and D. I. McCloskey, “Ankle stiffness of standing humans in response to imperceptible perturbations: reflex and task dependent components," J. Physiol., vol. 454, pp. 533-547, 1992.

[17] M. Rosenblum, G. Firsov, R. Kuuz, and B. Pompe, Human Postural Control: Force Plate Experiments and Modeling. Berlin: Springer, 1998, pp. 283-306.

[18] M. R. Popović, K. Nakazawa, I. P. I. Pappas, T. Keller, V. Dietz, and M. Morari, "Stability criteria for controlling standing in able-bodied subjects," J. Biomechan., vol. 33, no. 11, pp. 1359-1368, Nov. 2000.

[19] M. Mihelj, Z. Matjačić, and T. Bajd, "Postural activity of constrained subject in response to disturbance in sagittal plane," Gait and Posture, vol. 12, pp. 94-104, 2000.

[20] D. A. Winter, A. E. Patla, F. Prince, M. Ishac, and K. Gielo-Perczak, "Stiffness control of balance in quiet standing," J. Neurophysiol., vol. 80, no. 3, pp. 1211-1221, Sept. 1998.

[21] P. G. Morasso and M. Schieppati, "Can muscle stiffness alone stabilise upright standing?," J. Neurophysiol., vol. 82, no. 3, pp. 1622-1626, Sept. 1999 .

[22] M. Mihelj and M. Munih, "Minimum effort optimal control of ankle joints for unsupported standing in paraplegia," in Proc. 7th Ann. Conf. Int. Functional Electrical Stimulation Soc., Ljubljana, Slovenia, 2002, pp. 295-297.

[23] M. Mihelj and M. Munih, "Double inverted pendulum optimal control-basis for unsupported standing in paraplegia," in 7th Int. Workshop on Adv. Motion Contr., Maribor, Slovenia, 2000, pp. 121-126.

[24] M. Mihelj and M. Munih, "Unsupported standing with minimized ankle muscle fatigue," IEEE Trans. Biomed. Eng., 2003, to be published.

[25] Z. Matjačić, I. L. Johannesen, and T. Sinkjær, "A multi-purpose rehabilitation frame: a novel apparatus for balance training during standing of neurologically impaired individuals," J. Rehabil. Res. Develop., vol. 37, no. 6, pp. 681-691, 2000. 
[26] Z. Matjačić, "A multi-purpose rehabilitation frame: an apparatus experimental investigations of human balance and postural control," J. Med. Eng. Technol., vol. 24, pp. 250-254, 2000.

[27] R.-P. Jaime, "On the control of paraplegic standing using functional electrical stimulation," Ph.D. dissertation, Univ. of Glasgow, U.K., May 2002.

[28] G. F. Phillips, J. R. Adler, and S. J. G. Taylor, "A portable programmable eight-channel surface stimulator," in Proc. Ljubljana FES Conf., Ljubljana, Slovenia, 1993, pp. 166-168.

[29] L. Ljung, System Identification: theory for the user, Information and System Sciences Series, 1st ed. Englewood Cliffs, NJ: Prentice-Hall, 1987.

[30] K. J. Åström and B. Wittenmark, Computer Controlled Systems, 3th ed. Englewood Cliffs, NJ: Prentice-Hall, 1997.

[31] R.-P. Jaime, K. J. Hunt, and H. Gollee, "A polynomial $H_{\infty}$-controller for muscle moment control," in Proc. Europ. Contr. Conf. ECC'99, Karlsruhe, Germany, 1999.

[32] K. J. Hunt, R.-P. Jaime, and H. Gollee, "Robust control of electrically stimulated muscle using polynomial $H_{\infty}$-design," Contr. Eng. Practice, vol. 9, no. 3, pp. 313-328, 2001.

[33] R.-P. Jaime, Z. Matjačić, and K. J. Hunt, "Paraplegic standing supported by FES-controlled ankle stiffness," in Proc. 6th Ann. Conf. Int. Functional Electrical Stimulation Soc., Cleveland, OH, 2001.

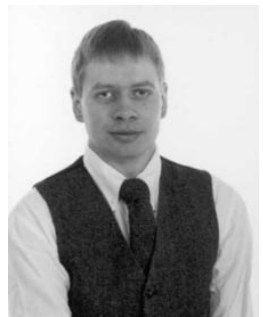

Ralf-Peter Jaime received the diploma degree in electrical engineering from the Technical University Berlin, Germany, in 1998 and the Ph.D. degree in control engineering from the University of Glasgow, Glasgow, U.K., in 2002. During his Ph.D. work, he was a student of Professor Kenneth J. Hunt working on the subject of paraplegic standing using FES.

$\mathrm{He}$ is currently with the Robert Bosch GmbH, Germany, working as a systems engineer on vehicle stability systems.

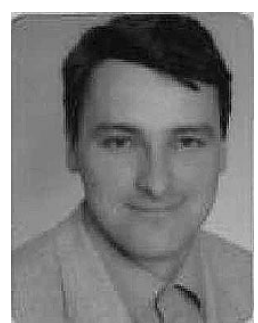

Zlatko Matjačić received the B.Sc., M.Sc., and D.Sc. degrees from the Faculty of Electrical Engineering, University of Ljubljana, Slovenia, in 1992, 1995, and 1998, respectively.

He was a Research and Teaching Assistant with the Faculty of Electrical Engineering, University of Ljubljana, from 1993 to 1998. From 1998 to 2001, he was an Assistant Professor of Biomedical Sciences and Engineering at Aalborg University, Aalborg, Denmark, and a Research Assistant at Jožef Stefan Institute, Ljubljana. Currently, he is an Assistant Director of Research with the Institute for Rehabilitation, Republic of Slovenia and an Assistant Professor of Biomechanics with the University College of Health Care, University of Ljubljana. He is also affiliated with the Jožef Stefan Institute where he holds a position of Research Associate. His research interests include human motion analysis and synthesis, biomechanics, control of human motion, orthotics and prosthetics, FES and development of biomechatronics devices for neurorehabilitation of human movement.

Dr. Matjačić received the prestigious The Jožef Stefan Golden Emblem Award for outstanding contributions made to science in his Doctoral theses.

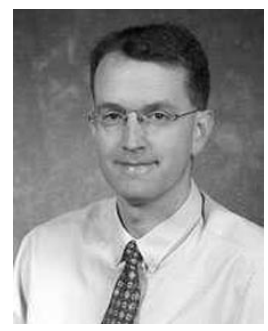

Kenneth J. Hunt is a professor of mechanical engineering and Director of the Centre for Rehabilitation Engineering at Glasgow University, Glasgow, Scotland, U.K. He is also a Clinical Research Professor at the National Spinal Injuries Unit of Glasgow's Southern General Hospital. The Centre for Rehabilitation Engineering (http://fesnet.eng.gla.ac.uk/CRE/) carries out basic and applied research in assistive technologies for function restoration. The principal focus of the work is on the control of functional electrical stimulation and its application in spinal cord injury rehabilitation. The main areas of current research are feedback control of balance in paraplegia; lower-limb cycling in paraplegia; and assisted upper-limb arm-cranking exercise in tetraplegia. 